\title{
Robust Model Predictive Control based on Gaussian Processes: Application to Drinking Water Networks
}

\author{
Ye Wang, Carlos Ocampo-Martinez and Vicenç Puig
}

\begin{abstract}
In this paper, a controller design based on robust Model Predictive Control (MPC) and Gaussian Processes (GP) for incorporating the disturbance forecasting has been proposed. Using a probabilistic system representation, the state trajectories considering the influence of disturbances can be obtained through the uncertainty propagation by using GP. Therefore, the worst-case state trajectories evolution over the MPC prediction horizon can be determined, which are potentially used by including them into the MPC cost function and constraints. For the purpose of inspecting the performance of proposed controller, it has been compared with a certainequivalent MPC and a chance-constrained MPC. Results of the application the proposed approach to Barcelona Drinking Water Network (DWN) have shown the effectiveness of the approach and comparison results with the other considered MPC approaches have shown the advantages and drawbacks of each approach.
\end{abstract}

\section{INTRODUCTION}

Robust Model Predictive Control (RMPC) is an interesting research field to deal with system disturbances and uncertainties. System disturbances and uncertainties can have a deep influence on the performance of a control system. Therefore, they should be properly considered into the MPC design. Model Predictive Control (MPC) with Gaussian Processes (GP) model was firstly introduced in [1], which proposes a new way of considering uncertainty in MPC. Thus, predictive control together with GP have also been considered for addressing the fault-tolerant problem [2]. Generally speaking, the GP approach is able to provide a new way of including the system disturbances in the predictive control framework.

In MPC, the system model is used to find an optimal control action along a prediction horizon according to the given control objectives and physical/operational constraints. Thus, a modelling approach shows to be necessary, which is not only able to consider system dynamics but also the dynamic effect of system disturbances. Hence, a proper disturbance forecasting approach should be used to produce reliable short-term forecasting results. Some forecasting approaches have been discussed in [3], [4]. After applying a disturbance forecasting approach, the forecasting results will be incorporated into the optimal control input computation.

In this paper, the main contribution consists in the design of an RMPC controller based on GP by utilizing the disturbance forecasting results from the approach in [4], which is called in the sequel $D S H W-G P$. The proposed approach combines the double-seasonal Holt-Winters (DSHW)

Y. Wang, C. Ocampo-Martinez and V. Puig are with Institut de Robòtica i Informàtica Industrial (CSIC-UPC), Technical University of Catalonia, C/. Llorens i Artigas, 4-6. 08028 Barcelona, Spain. E-mail: \{ywang, cocampo, vpuig\}airi.upc.edu. method and GP regression algorithms in order to propagate the disturbance forecast uncertainty into the system states. Therefore, the RMPC controller has been designed by considering the worst-case situation of system states in order to achieve robustness against uncertainty. DSHW-GP is a mixed methodology that incorporates advantages from both the DSHW and GP algorithms. Unlike traditional forecasting approaches, e.g., [5], [6], DSHW-GP is useful for being integrated with GP since it allows to model the uncertain part of the demand forecast in the GP framework.

Moreover, for the sake of testing the effectiveness and efficiency of the proposed control strategy, two MPC strategies are also briefly introduced: the certainty-equivalent MPC (CE-MPC) and the chance-constrained MPC (CC-MPC) [7]. A case study based on the Barcelona Drinking Water Network (DWN) has been considered, where the aforementioned MPC strategies are applied and compared through some given key performance indicators (KPI). Notice that the water demand can be treated as the system disturbance in a DWN. Obtained results have shown the advantages and disadvantages for all the considered MPC techniques.

The reminder of this paper is organized as follows. In Section II, the problem formulation is presented. The RMPC based on GP approach is presented in Section III. In Section IV, the application of the considered approaches to Barcelona DWN is described, while simulation results are shown in Section V. Finally, the main conclusions are drawn in Section VI.

\section{Problem Statement}

\section{A. Control-oriented Model}

Consider that the system to be controlled can be described by means of a discrete-time control-oriented model

$$
\mathbf{x}_{k+1}=\mathbf{A} \mathbf{x}_{k}+\mathbf{B} \mathbf{u}_{k}+\mathbf{d}_{k},
$$

where $\mathbf{x}$ and $\mathbf{u}$ denote system state and control input vectors, respectively. Moreover, A, B are the system matrices of suitable dimensions, $\mathbf{d}_{k}$ represents the vector of system disturbances and $k$ is the discrete time.

The components of an RMPC-based closed loop are shown in Fig. 1. There, the system is affected by the influence of external disturbances, which should be properly forecasted facing their inclusion in the prediction model used by the MPC controller. Notice that the loop is closed by using an observer, which can be seen as a output-feedback scheme. This paper considers that the observer does not consider the effect of the disturbances. 


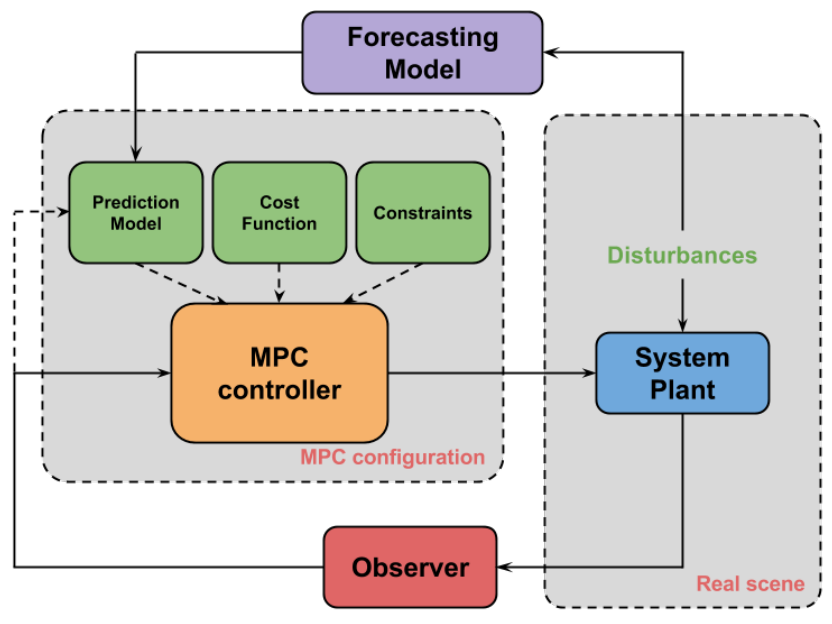

Fig. 1. Components of an RMPC closed-loop topology

\section{B. Disturbance Forecasting Approach}

According to (1), system states are influenced by the exogenous disturbances. As stated before, the disturbance forecasting approach considered in this paper is the DSHWGP, a probabilistic approach reported in [4]. The forecasting results are not a set of deterministic values but a set of probabilities that can be described as

$$
\mathbf{d}_{k} \sim \mathcal{N}\left(\overline{\mathbf{d}}_{k}, \Sigma_{\mathbf{d}_{k}}\right)
$$

where $\mathcal{N}$ denotes normal distribution, $\overline{\mathbf{d}}_{k}$ and $\Sigma_{\mathbf{d}_{k}}$ correspond to the mean and variance disturbance estimates, which are used for the RMPC controller design.

\section{Generalized RMPC}

From (2), the prediction model related to the MPC design is written in a probabilistic way as

$$
\mathbf{x}_{k} \sim \mathcal{N}\left(\overline{\mathbf{x}}_{k}, \Sigma_{\mathbf{x}_{k}}\right)
$$

where $\overline{\mathbf{x}}_{k}, \Sigma_{\mathbf{x}_{k}}$ are the mean and the variance of the system states $\mathbf{x}_{k}$. This paper addresses the optimization problem behind the MPC design with two probabilistic models: (i) the probabilistic representation of the state-space model of the network and (ii) the forecasting model of the disturbances. These models are used to find a sequence of control actions over a given prediction horizon $H_{p} \in \mathbb{N}_{+}$. The receding horizon method is used for selecting the first control action as the optimal one at each time instant. In general, a basic RMPC controller is formulated by solving the multi-objective finite horizon optimization problem (FHOP) in Problem 1.

Problem 1 (Generalized FHOP):

$$
\min _{u_{k}} \mathcal{J} \triangleq \mathbb{E}\left[\sum_{n=1}^{\mathcal{O}} \sum_{i=0}^{H_{p}-1} \lambda_{n} \mathcal{J}_{n}\left(\mathbf{x}_{k+i+1 \mid k}, \mathbf{u}_{k+i \mid k}, \mathbf{d}_{k+i \mid k}\right)\right],
$$

subject to:

$$
\begin{gathered}
\mathbf{x}_{k+i+1 \mid k} \sim \mathcal{N}\left(\overline{\mathbf{x}}_{k+i+1 \mid k}, \Sigma_{\mathbf{x}_{k+i+1 \mid k}}\right) \\
\mathbf{d}_{k+i \mid k} \sim \mathcal{N}\left(\overline{\mathbf{d}}_{k+i \mid k}, \Sigma_{\mathbf{d}_{k+i \mid k}}\right) \\
\mathbf{u}_{\min } \leq \mathbf{u}_{k+i \mid k} \leq \mathbf{u}_{\max } \\
\mathbf{x}_{\min } \leq \mathbf{x}_{k+i+1 \mid k} \leq \mathbf{x}_{\max } \\
\left(\mathbf{x}_{k \mid k}, \mathbf{d}_{k \mid k}\right)=\left(\mathbf{x}_{k}, \mathbf{d}_{k}\right),
\end{gathered}
$$

where $\mathcal{O} \in \mathbb{N}_{+}$is the total number of control objectives $\mathcal{J}_{n}$ that conform the whole cost function $\mathcal{J}, \mathbb{E}$ is the expectation operator, $\mathcal{N}$ is the normal distribution with the proper mean and variance variables, together with mean parameters $\overline{\mathbf{x}}_{k+i+1 \mid k}, \bar{d}_{k+i \mid k}$ and variance parameters $\Sigma_{\mathbf{x}_{k+i+1 \mid k}}$, $\Sigma_{\mathbf{d}_{k+i+1 \mid k}}$.

\section{Robust Model Predictive Control based on GaUSSIAN PROCESSES}

\section{A. Uncertainty Propagation of System States}

By using the proposed DSHW-GP approach, the forecasting disturbances over the MPC prediction horizon are obtained as

$$
\mathbf{d}_{k+i} \sim \mathcal{N}\left(\overline{\mathbf{d}}_{k+i}, \Sigma_{\mathbf{d}_{k+i}}\right), \quad i=0,1,2, \ldots, H_{p}-1,
$$

where $\overline{\mathbf{d}}_{k+i}$ is the mean estimation and $\Sigma_{\mathbf{d}_{k+i}}$ is the variance estimation, both at time instant $k+i$.

Under a probabilistic representation of a control system, the prediction model can be written down as $p\left(\mathbf{x}_{i+1}\right) \sim$ $\mathcal{N}\left(\overline{\mathbf{x}}_{k+1}, \Sigma_{\mathbf{x}_{k+1}}\right)$ and the state transition probability can be approximated by

$$
\left(\mathbf{x}_{k}, \mathbf{x}_{k+1} \mid \overline{\mathbf{x}}_{k}, \Sigma_{\mathbf{x}_{k}}\right) \sim \mathcal{N}\left(\left[\begin{array}{c}
\overline{\mathbf{x}}_{k} \\
\overline{\mathbf{x}}_{k+1}
\end{array}\right],\left[\begin{array}{cc}
\Sigma_{\mathbf{x}_{k}} & \Sigma_{\mathbf{x}_{k}, \mathbf{x}_{k+1}} \\
\Sigma_{\mathbf{x}_{k}, \mathbf{x}_{k+1}}^{T} & \Sigma_{\mathbf{x}_{k+1}}
\end{array}\right]\right),
$$

where $\overline{\mathbf{x}}_{k}, \overline{\mathbf{x}}_{k+1}$ represent the mean of states at two consecutive steps and $\Sigma_{\mathbf{x}_{k}}, \Sigma_{\mathbf{x}_{k+1}}$ represent the corresponding variance matrices. Moreover, $\Sigma_{\mathbf{x}_{k}, \mathbf{x}_{k+1}}$ denotes the covariance matrix of states at time instant $k$ and $k+1$. Since the system model in (1) is linear, (7) can be simplified as

$$
\left[\begin{array}{c}
\mathbf{x}_{k} \\
\mathbf{x}_{k+1}
\end{array}\right] \sim \mathcal{N}\left(\left[\begin{array}{c}
\overline{\mathbf{x}}_{k} \\
\overline{\mathbf{x}}_{k+1}
\end{array}\right],\left[\begin{array}{cc}
\Sigma_{\mathbf{x}_{k}} & \Sigma_{\mathbf{x}_{k}, \mathbf{x}_{k+1}} \\
\Sigma_{\mathbf{x}_{k}, \mathbf{x}_{k+1}}^{T} & \mathbf{A} \Sigma_{\mathbf{x}_{k}} \mathbf{A}^{T}+\Sigma_{\mathbf{d}_{k}}
\end{array}\right]\right) .
$$

Thus, both the mean and the variance of the system states are written as

$$
\begin{aligned}
\overline{\mathbf{x}}_{k+1} & =\mathbf{A} \overline{\mathbf{x}}_{k}+\mathbf{B} \mathbf{u}_{k}+\overline{\mathbf{d}}_{k}, \\
\Sigma_{\mathbf{x}_{k+1}} & =\mathbf{A} \Sigma_{\mathbf{x}_{k}} \mathbf{A}^{T}+\Sigma_{\mathbf{d}_{k}} .
\end{aligned}
$$

Then, the confidence interval (CI) of the system states over $H_{p}$ can be written as

$$
\mathbf{x}_{k+i} \in\left[\overline{\mathbf{x}}_{k+i}-\alpha \Sigma_{x_{k+i}}^{1 / 2}, \overline{\mathbf{x}}_{k+i}+\alpha \Sigma_{\mathbf{x}_{k+i}}^{1 / 2}\right],
$$

with $i=1, \ldots, H_{p}$, where $\alpha$ is a critical value corresponding to a confidence level in order to decide the width of the CI. Common choices of a confidence level are $95 \%$ or $98 \%$. The relation between the critical value and the confidence level is given by

$$
\alpha=\Phi^{-1}\left(1-\frac{l}{2}\right),
$$


where $l$ denotes the confidence level and $\Phi^{-1}$ represents the inverse standard probability density function.

The upper and lower bounds of system states are growing over the prediction horizon. The worst-case state evolutions are included into the MPC constraints as follows:

$$
\begin{aligned}
& \mathbf{x}_{\min } \leq \overline{\mathbf{x}}_{k}+\alpha \Sigma_{x_{k}}^{1 / 2} \leq \mathbf{x}_{\max } \\
& \overline{\mathbf{x}}_{k}-\alpha \Sigma_{\mathbf{x}_{k}}^{1 / 2} \geq \mathbf{x}_{S}
\end{aligned}
$$

where $\overline{\mathbf{x}}_{k}+\alpha \Sigma_{x_{k}}^{1 / 2}$ is the upper bound of states, which should be lower than $\mathbf{x}_{\max }$. Moreover, $\overline{\mathbf{x}}_{k}-\alpha \Sigma_{\mathbf{x}_{k}}^{1 / 2}$ corresponds with the lower bound of the states, which should be greater than an assumed safety amount $\mathbf{x}_{S}$. Notice that $\mathbf{x}_{S}$ is required to guarantee the water supply in exceptional situations. Here, the worst-case lower bound of the states should be kept greater than the safety amount defined for each state.

With all the latter considerations, Problem 1 can be reformulated as follows.

\section{Problem 2 (Improved GP-MPC):}

$$
\min _{u_{k}} \mathcal{J}_{k} \triangleq \mathbb{E}\left[\sum_{n=1}^{\mathcal{O}} \sum_{i=0}^{H_{p}-1} \lambda_{n} \mathcal{J}_{n}\left(\mathbf{x}_{k+i+1 \mid k}, \mathbf{u}_{k+i \mid k}, \mathbf{d}_{k+i \mid k}\right)\right] \text {, }
$$

subject to:

$$
\begin{array}{r}
\overline{\mathbf{x}}_{k+i+1 \mid k}=\mathbf{A} \overline{\mathbf{x}}_{k+i \mid k}+\mathbf{B} \mathbf{u}_{k+i \mid k}+\overline{\mathbf{d}}_{k+i \mid k} \\
\Sigma_{\mathbf{x}_{k+i+1 \mid k}}=A \Sigma_{\mathbf{x}_{k+i \mid k}} A^{T}+\Sigma_{\mathbf{d}_{k+i \mid k}} \\
\mathbf{u}_{\min } \leq \mathbf{u}_{k+i+1 \mid k} \leq \mathbf{u}_{\max } \\
\mathbf{x}_{\min } \leq \overline{\mathbf{x}}_{k+i \mid k}+\alpha \Sigma_{x_{k+i \mid k}}^{1 / 2} \leq \mathbf{x}_{\max } \\
\overline{\mathbf{x}}_{k+i \mid k}-\alpha \Sigma_{\mathbf{x}_{k+i \mid k}}^{1 / 2} \geq \mathbf{x}_{S} \\
\left(\mathbf{x}_{k \mid k}, \mathbf{d}_{k \mid k}\right)=\left(\mathbf{x}_{k}, \mathbf{d}_{k}\right)
\end{array}
$$

\section{Case Study: Barcelona Drinking Water NEWTWORK}

\section{A. System Description}

The Barcelona DWN supplies $237.7 \mathrm{hm}^{3}$ of drinking water to approximately three million consumers every year, covered $424 \mathrm{~km}^{2}$ area. The entire network is composed of 63 storage tanks, three surface sources, seven underground sources, 79 pumps, 50 valves, 18 nodes and 88 water demands shown in Figure 2. Currently, AGBAR ${ }^{1}$ is in charge of managing the entire network through a supervisory control system. It supplies potable water to the Metropolitan Area of Barcelona (Catalonia, Spain). The main water resources come from rivers Llobregat, Ter and Besòs, with 52\%, 46\% and $2 \%$ of the total water supply, respectively. These sources are regulated by dams that have an overall capacity of 600 cubic hectometres.

\footnotetext{
${ }^{1}$ AGBAR: Aguas de Barcelona, S. A. Company which manages the drinking water transport and distribution in Barcelona (Spain).
}

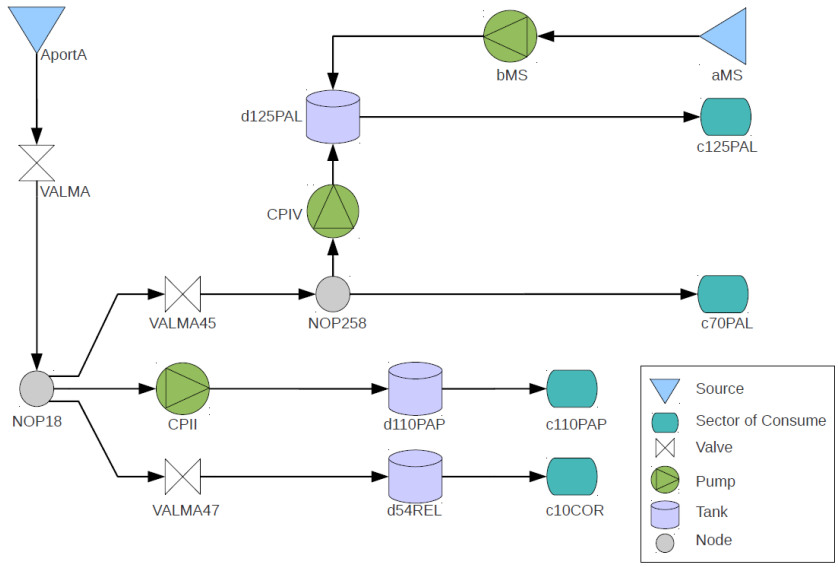

Fig. 2. Portion of the Barcelona DWN used as case study

Fig. 2 shows a representative portion of the Barcelona DWN selected as the case study of this paper. This DWN part includes three water storage tanks, three pumps, three valves and four water demand sectors. The mathematical model of the case study as well as other mathematical features can be found in [8].The parameters of the model have been adjusted by using real data provided by the DWN management company.

\section{B. Control-oriented Model of Drinking Water Networks}

The control-oriented model of DWN is described by the following set of linear discrete difference-algebraic equations for all time instant $k \in \mathbb{N}$ [7]:

$$
\begin{aligned}
\mathbf{x}_{k+1} & =\mathbf{A} \mathbf{x}_{k}+\mathbf{B} \mathbf{u}_{k}+\mathbf{B}_{d} \mathbf{d}_{k}, \\
0 & =\mathbf{E}_{u} \mathbf{u}_{k}+\mathbf{E}_{d} \mathbf{d}_{k},
\end{aligned}
$$

where $\mathbf{x}_{k}, \mathbf{u}_{k}, \mathbf{d}_{k}$ denote the state vector, the manipulated flows through actuators and the demanded flow as additive measured disturbances, respectively. Pumps and valves are considered as the actuators. Moreover, (15a) describes the dynamics of storage tanks while (15b) presents the static relations within the DWN at network nodes. Matrices $\mathbf{A}$, $\mathbf{B}, \mathbf{B}_{d}, \mathbf{E}_{u}$ and $\mathbf{E}_{d}$ are obtained from the network topology (see [8], for the numerical values of the part of the network considered in this paper).

For the purpose of incorporating disturbance forecasting results into system states by the GP, the standard controloriented model (1) is needed. A compact form of (15) can be written as

$$
\mathbf{x}_{k+1}=\mathbf{A} \mathbf{x}_{k}+\tilde{\mathbf{B}} \tilde{\mathbf{u}}_{k}+\tilde{\mathbf{B}}_{p} \mathbf{d}_{k},
$$

where $\tilde{\mathbf{u}}_{k}$ is the reduced vector control inputs expressed as

$$
\mathbf{u}_{k}=\tilde{\mathbf{P}} \tilde{\mathbf{M}}_{1} \tilde{\mathbf{u}}_{k}+\tilde{\mathbf{M}}_{2} \mathbf{d}_{k},
$$

with $\tilde{\mathbf{B}}, \tilde{\mathbf{B}} p, \tilde{\mathbf{P}}, \tilde{\mathbf{M}}_{1}, \tilde{\mathbf{M}}_{2}$ are calculation matrices with suitable dimensions. The detailed calculation can be found in [7]. 
According to (9), the prediction model of the DWN can be written as

$$
\begin{aligned}
\overline{\mathbf{x}}_{k+1} & =\mathbf{A} \overline{\mathbf{x}}_{k}+\widetilde{\mathbf{B}} \mathbf{u}_{k}+\widetilde{\mathbf{B}}_{p} \overline{\mathbf{d}}_{k}, \\
\Sigma_{\mathbf{x}_{k+1}} & =\mathbf{A} \Sigma_{\mathbf{x}_{k}} \mathbf{A}^{T}+\widetilde{\mathbf{B}}_{p} \Sigma_{\mathbf{d}_{k}} \widetilde{\mathbf{B}}_{p}^{T} .
\end{aligned}
$$

\section{Management Criteria of Drinking Water Network}

The operational goals in the management of the DWN are economic, safety, smoothness, and are respectively stated as follows [7], [9]:

1) Provide a reliable water supply minimising water production and water transport costs.

2) Guarantee the availability of enough water in each storage tank to satisfy its underlying stochastic demand.

3) Operate the DWN under smooth control actions.

\section{Constraints}

Since some manipulated variables $\mathbf{u}_{k}$ have relationships with some water demands, the constraint for reduced manipulated variable $\widetilde{\mathbf{u}}_{k}$ should maintain the constraint for the full manipulated variables $u_{k}$. Hence,

$$
\mathbf{u}_{\min } \leq \tilde{\mathbf{P}} \tilde{\mathbf{M}}_{1} \tilde{\mathbf{u}}_{k}+\tilde{\mathbf{M}}_{2} \mathbf{d}_{k} \leq \mathbf{u}_{\max }
$$

where $\mathbf{u}_{\min }$ and $\mathbf{u}_{\max }$ are physical limitations of the manipulated variables $\mathbf{u}_{k}$.

On the other hand, the system states are also bounded according to the following expressions:

$$
\begin{gathered}
\mathbf{x}_{\min } \leq \mathbf{x}_{k} \leq \mathbf{x}_{\max } \\
\mathbf{x}_{k} \geq \vartheta_{\text {net }, k}
\end{gathered}
$$

where $\mathbf{x}_{\min }$ and $\mathbf{x}_{\max }$ are limitations of water storage tanks. $\vartheta_{\text {net }, k}$ represents net demands at time instant $k$ which can be decomposed into two parts, the endogenous demands from neighbouring tanks or nodes $\mathbf{B}_{e} \mathbf{u}_{k}$ and the exogenous demands from consumer sectors $\mathbf{B}_{p} \mathbf{d}_{k}$. Hence, $\vartheta_{\text {net, } k}$ can be computed as

$$
\vartheta_{\text {net }, k}=\left|\mathbf{B}_{e} \mathbf{u}_{k}+\mathbf{B}_{p} \mathbf{d}_{k}\right| .
$$

\section{E. Objective Functions}

According to safety criteria, (20b) can be set as a soft constraint in the following way:

$$
\mathbf{x}_{k} \geq \vartheta_{\text {net }, k}-\xi_{k} \geq \mathbf{0} \quad \forall k,
$$

where $\xi_{k} \in \mathbb{R}^{n_{x}}$ represents the amount of volume in $\mathrm{m}^{3}$ that goes down from the desired safety thresholds. Then, this approach introduces a new performance indicator to be minimised, defined as

$$
\mathcal{J}_{S, k} \triangleq\left\|\xi_{k}\right\|_{2, \mathbf{w}_{x}}^{2} .
$$

Regarding the first and third management criterion, they can be mathematically formulated as follows

$$
\begin{aligned}
\mathcal{J}_{E, k} & \triangleq\left\|\left(\alpha_{1}+\alpha_{2, k}\right)^{\mathrm{T}} \mathbf{u}_{k}\right\|_{1, \mathbf{w}_{e}}, \\
\mathcal{J}_{\Delta U, k} & \triangleq\left\|\Delta \mathbf{u}_{k}\right\|_{2, \mathbf{w}_{u}}^{2},
\end{aligned}
$$

where $\mathcal{J}_{E} \in \mathbb{R}$ represents the economic cost of network operation taking into account water production cost $\alpha_{1} \in$
$\mathbb{R}^{n_{u}}$ and water pumping cost $\alpha_{2} \in \mathbb{R}^{n_{u}}$, which changes every time instant $k$ according to the variable electric tariff; $\mathcal{J}_{\Delta U} \in \mathbb{R}$ represents the penalisation of control signal variations $\Delta \mathbf{u}_{k} \triangleq \mathbf{u}_{k}-\mathbf{u}_{k-1}$, to extend actuators life and assure a smooth operation; $\|\cdot\|_{p, \mathbf{W}}$ is a weighted $p$-norm; and $\mathbf{W}_{e}, \mathbf{W}_{u}$ are diagonal matrices that weight each decision variable in the corresponding control objective.

\section{F. FHOP for DWNS}

The predication horizon $H_{p}$ is equal to 24 with a control sampling time of one hour. These values are selected taking into account the seasonality of the demand and the dynamics of the network. The control objectives are prioritised through weights $\lambda_{1}, \lambda_{2}, \lambda_{3}$ and aggregated in the performance index. The GP-MPC law for the DWN consists in solving a FHOP given in Problem 3.

Problem 3 (GP-MPC for DWN):

$$
\begin{aligned}
\min _{u_{k}} \mathcal{J}_{k} \triangleq & \left\{\lambda_{1} \sum_{i=0}^{H_{p}-1} \mathcal{J}_{E, k+i \mid k}+\lambda_{2} \sum_{i=0}^{H_{p}-1} \mathcal{J}_{S, k+i \mid k}\right. \\
& \left.+\lambda_{3} \sum_{i=0}^{H_{p}-1} \mathcal{J}_{\Delta U, k+i \mid k}\right\}
\end{aligned}
$$

subject to:

$$
\begin{gathered}
\overline{\mathbf{x}}_{k+i+1 \mid k}=\mathbf{A} \overline{\mathbf{x}}_{k+i \mid k}+\widetilde{\mathbf{B}} \widetilde{\mathbf{u}}_{k+i \mid k}+\widetilde{\mathbf{B}}_{p} \overline{\mathbf{d}}_{k+i \mid k} \\
\Sigma_{\mathbf{x}_{k+i+1 \mid k}}=A \Sigma_{\mathbf{x}_{k+i \mid k}} A^{T}+\widetilde{\mathbf{B}}_{p} \Sigma_{\mathbf{d}_{k+i \mid k}} \widetilde{\mathbf{B}}_{p}^{T} \\
\mathbf{u}_{\min } \leq \widetilde{\mathbf{u}}_{k+i+1 \mid k} \leq \mathbf{u}_{\max } \\
\mathbf{x}_{\min } \leq \overline{\mathbf{x}}_{k+i \mid k}+\alpha \Sigma_{x_{k+i \mid k}}^{1 / 2} \leq \mathbf{x}_{\max } \\
\overline{\mathbf{x}}_{k+i \mid k}-\alpha \Sigma_{\mathbf{x}_{k+i \mid k}}^{1 / 2} \geq \mathbf{x}_{S}-\xi_{k+1 \mid k} \geq 0 \\
\left(\mathbf{x}_{k \mid k}, \mathbf{d}_{k \mid k}\right)=\left(\mathbf{x}_{k}, \mathbf{d}_{k}\right)
\end{gathered}
$$

\section{Simulation Results}

\section{A. Key Performance Indicators}

For the purpose of comparing obtained results, three KPIs have been defined based on the DWN objective function and the control objectives as discussed below [7].

1) Economic KPI: The economical performance indicator is related with the water transportation costs, which is defined as

$$
K P I_{E} \triangleq \frac{1}{n_{s}} \sum_{k=1}^{n_{s}}\left|\left(\alpha_{1}+\alpha_{2, k}\right) \mathbf{u}_{k}\right| \Delta t,
$$

where $n_{s}$ is the number of hours considered in the assessment and $\Delta t$ is sampling time that is equal to $3600 \mathrm{~s}$ ( 1 hour) in this case study. Moreover, $\alpha_{1}$ and $\alpha_{2, k}$ are the water production cost and hourly electric cost with respect to control action $u_{k}$ at time instant $k$, respectively.

2) Safety KPI: The safe performance indicator is related to volume-regulation strategy of tanks in the DWN, which is defined as

$$
K P I_{S} \triangleq \frac{1}{n_{s}} \sum_{k=1}^{n_{s}} \sum_{i=1}^{n_{x}} \xi_{(i), k}
$$




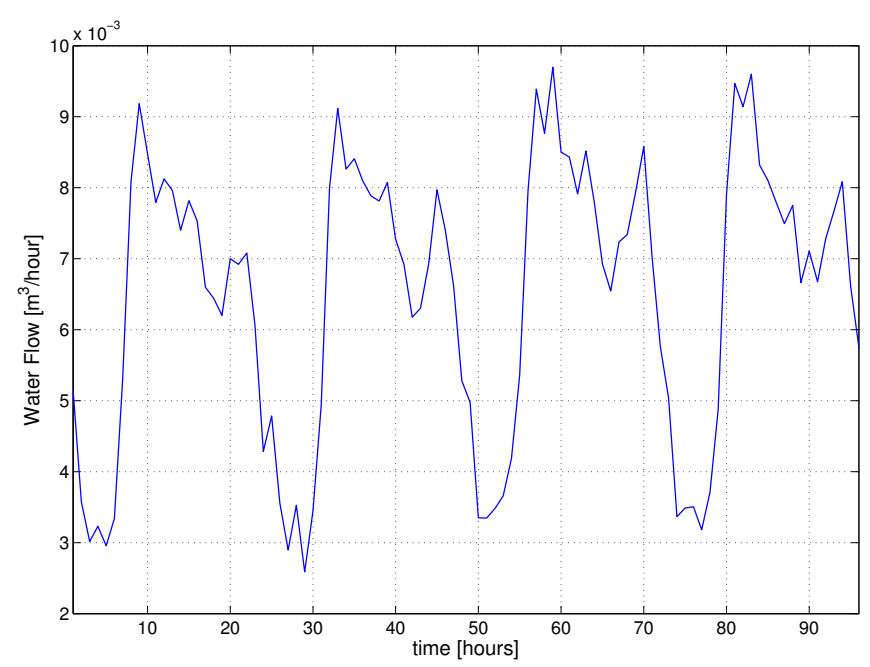

Fig. 3. Experimental data of consume Demands

where $n_{s}$ is the horizon of the assessment while $n_{x}$ is the number of storage tanks and $\xi_{(i), k} \triangleq\left|x_{(i), k}-x_{s_{(i), k}}\right|$ is the level of violation of the soft constraint for the $i^{t h}$ tank at time instant $k$.

3) Smoothness KPI: The smooth performance indicator is in terms of switch frequency of control actions, which is defined as

$$
K P I_{\Delta U} \triangleq \frac{1}{n_{s}} \sum_{k=1}^{n_{s}} \sum_{i=1}^{n_{u}}\left(\Delta \mathbf{u}_{(i), k}\right)^{2}
$$

where $n_{s}$ is the horizon of the assessment while $n_{u}$ is the number of actuators. Moreover, $\Delta \mathbf{u}_{(i), k}$ is the variation of the control action for the $i^{\text {th }}$ actuator at the time instant $k$, which is computed by $\Delta \mathbf{u}_{(i), k}=\mathbf{u}_{(i), k}-\mathbf{u}_{(i), k-1}$.

\section{B. Results}

All numerical simulations were performed in Matlab R2012b ${ }^{\circledR}$ 64-bit, running in a PC with CPU of Intel ${ }^{\circledR}$ Core $^{\mathrm{TM}} \mathrm{i5}-3210 \mathrm{M} @ 2.50 \mathrm{GHz}$ and RAM of 4GB. The experimental data of consume demands are synthetic with considering some certain noise, for instance variance of $5 \%$ of total demand. A sampled experimental water demand data are plotted in the Fig. 3.

For the Problem 3, the closed-loop system has been simulated with different selections of confidence level. The CE-MPC and CC-MPC problems have been considered with the same objective functions than Problem 3. The weights for economic, safety and smoothing are 100, 10 and 0.005, respectively.

1) Results of GP-MPC for DWN: The simulation has been carried out over a time period of four days (96 hours) with a sampling time of one hour. The water demand forecasts are obtained from using DSHW-GP algorithm with training data of the past seven months.

Fig. 4 shows control actions, system state and water demands for the tank 110PAP over the simulation period of 96 hours, respectively. The control actions are varying smoothly as the water price fluctuations. The water are accumulated
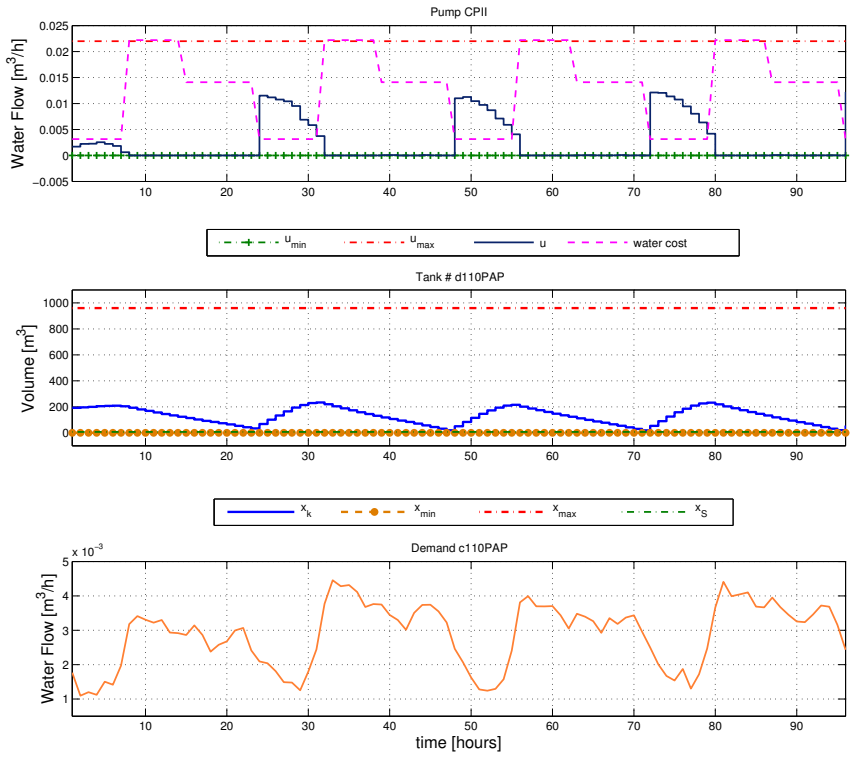

Fig. 4. Simulation Results of the GP-MPC with confidence level of $95 \%$

during the water price is low. The middle plot in Fig. 4 shows the water level in the tank, which is always above its safety amount considering the existing system disturbances. The last plot shows the water demands over the four days, which have potential daily pattern with some disturbances. Different confidence levels have been considered. With these options, the simulations have been run. Table I shows the KPIs for each case.

TABLE I

COMPARISONS OF GP-MPC FOR BARCELONA DWN WITH DIFFERENT CONFIDENCE LEVELS.

\begin{tabular}{lccc}
\hline Case & $\mathrm{KPI}_{E}$ & $\mathrm{KPI}_{S}$ & $\mathrm{KPI}_{\Delta U}$ \\
\hline GP-MPC $@ 98 \%$ & 27.1002 & $8.8390 \mathrm{e}+04$ & $1.2757 \mathrm{e}-06$ \\
GP-MPC $_{995 \%}$ & 27.1070 & $8.8175 \mathrm{e}+04$ & $1.2757 \mathrm{e}-06$ \\
GP-MPC $_{9} 90 \%$ & 27.0972 & $8.8076 \mathrm{e}+04$ & $1.2757 \mathrm{e}-06$ \\
GP-MPC $_{980 \%}$ & 27.0922 & $8.7780 \mathrm{e}+04$ & $1.2757 \mathrm{e}-06$ \\
\hline
\end{tabular}

In Table I, as the confidence level increases, $\mathrm{KPI}_{E}$ and $\mathrm{KPI}_{S}$ are decreasing and $\mathrm{KPI}_{\Delta U}$ is merely changing. That means the control system is safer but involves more costs. This is reasonable since there is a trade-off between water supply guarantees and minimizing the economic operation.

2) Comparisons with CE-MPC and CC-MPC for DWN:

Both CE-MPC and CC-MPC strategies have been applied to this case study with the same initial conditions as the GP-MPC proposed approach. The details of these two approaches applied to this case study can be found in [8] and [7], respectively.

Table II shows calculations of KPIs of each MPC strategy. The economic KPI of GP-MPC and CC-MPC are similar and higher than of CE-MPC because they considered the uncertainty associated with the demand forecast. 
TABLE II

COMPARISONS OF THREE MPC STRATEGIES FOR BARCELONA DWN BY USING KPIS

\begin{tabular}{cccc}
\hline Approach & $\mathrm{KPI}_{E}$ & $\mathrm{KPI}_{S}$ & $\mathrm{KPI}_{\Delta U}$ \\
\hline GP-MPC & 26.9749 & $7.8835 \mathrm{e}+04$ & $1.2757 \mathrm{e}-06$ \\
CE-MPC & 26.9480 & $7.7762 \mathrm{e}+04$ & $1.2757 \mathrm{e}-06$ \\
CC-MPC & 26.9673 & $7.8015 \mathrm{e}+04$ & $1.2757 \mathrm{e}-06$ \\
\hline
\end{tabular}

With the purpose of proving the equivalence of GP-MPC and CC-MPC with equivalent selection of confidence level, both of $\delta_{x}$ and $\delta_{d}$ were selected as 0.05 . Meanwhile $\alpha$ for the GP-MPC selects the average of $\alpha_{x}$ and $\alpha_{d}$ with the same selections of $\delta_{x}$ and $\delta_{d}$. Hence, $\alpha$ is 3.2944. The CE-MPC are also executed with the same demand forecasts. All results are shown in Table III.

TABLE III

RELATIONSHIPS BETWEEN RISK TERMS AND CRITICAL VALUES

\begin{tabular}{cccc}
\hline$\delta_{x}$ & $\delta_{d}$ & $\alpha_{x}$ & $\alpha_{d}$ \\
\hline 0.01 & 0.01 & 3.8101 & 3.6352 \\
0.05 & 0.05 & 3.3918 & 3.1970 \\
0.10 & 0.10 & 3.1970 & 2.9913 \\
\hline
\end{tabular}

Fig. 5 shows the comparison between both the GC-MPC and CC-MPC approaches, in which the curves show a slight difference on the way the flows are computed. Fig. 6 shows the system state evolutions over the simulation time. For the state evolutions of the tank $d 54 R E L$, it is clear that results of GP-MPC and CC-MPC are approximately equivalent.

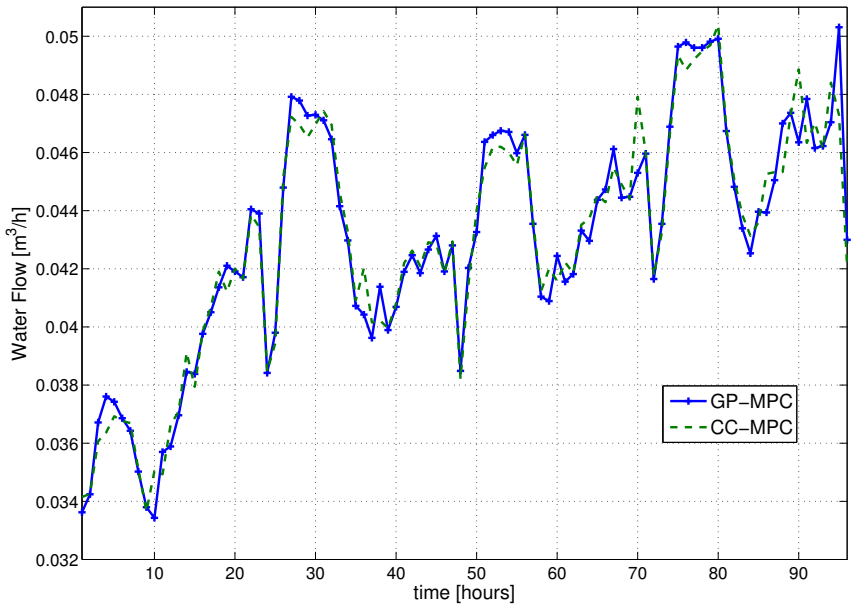

Fig. 5. Comparisons of selected control actions of three approaches

\section{CONCLUSIONS}

This paper has developed a new RMPC approach which is called GP-MPC strategy to manage system disturbances. For the MPC strategy, the prediction has to be executed considering system disturbances. By using DSHW-GP algorithm, the system disturbances have been forecasted over the MPC prediction horizon. The effectiveness of GP-MPC strategy has been shown through a case study by incorporating forecasted

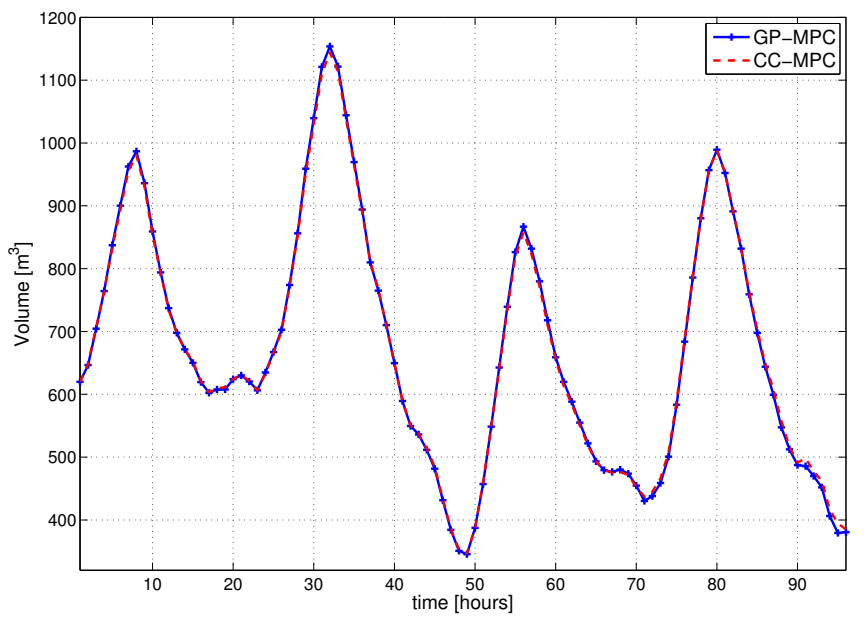

Fig. 6. Comparisons of selected system states of three approaches

water demands to system states and considering worst-case state evolution with disturbances. The simulation results have been shown and discussed. The proposed GP-MPC strategy has been compared with CE-MPC and CC-MPC. The results prove out that GP-MPC and CC-MPC are equivalent when the initial conditions are quite similar. Compared with CEMPC that ignores the existence of system disturbances, both GP-MPC and CC-MPC are able to ensure the system is still running with effects from system disturbances.

\section{ACKNOWLEDGEMENTS}

This work has been partially supported by the projects ECOCIS (DPI-2013-48243-C2-1-R) and EFFINET (FP7ICT-2012-318556).

\section{REFERENCES}

[1] J. Kocijan, R. Murray-Smith, C. Rasmussen, and B. Likar, "Predictive control with gaussian process models," EUROCON 2003. Computer as a Tool. The IEEE Region 8, vol. 1, pp. 352-356, 2003.

[2] J. M. Maciejowski and X. Yang, "Fault tolerant control using gaussian processes and model predictive control," in 2013 Conference on Control and Fault-Tolerant Systems (SysTol), Nice, 2013, pp. 1-12.

[3] J. Blanch, J. Quevedo, J. Saludes, and V. Puig, "Short-term demand forecasting for operational control of the barcelona water transport network," in Conferencia Nacional de Jóvenes Profesionales del Agua de España, Barcelona, 2010.

[4] Y. Wang, C. Ocampo-Martínez, V. Puig, and J. Quevedo, "Gaussianprocess-based demand forecasting for predictive control of drinking water networks," in 9th International Conference on Critical Information Infrastructures Security, Limassol, Cyprus, 2014.

[5] W. R. Christiaanse, "Short-term load forecasting using general exponential smoothing," IEEE Transactions on Power Apparatus and Systems, vol. 90, no. 2, pp. 900-911, 1971.

[6] P. J. Harrison, "Exponential smoothing and short-term sales forecasting," Management Science, vol. 13, no. 11, pp. 821-842, 1967.

[7] J. M. Grosso, C. Ocampo-Martínez, V. Puig, and B. Joseph, "Chanceconstrained model predictive control for drinking water networks," Journal of Process Control, vol. 24, no. 5, pp. 504-516, 2014.

[8] J. Grosso, C. Ocampo-Martinez, V. Puig, D. Limon, and M. Pereira, "Economic MPC for the management of drinking water networks," in Proceedings of the European Control Conference, Strasburg (France), 2014, pp. 1-12.

[9] C. Ocampo-Martínez, V. Puig, G. Cembrano, and J. Quevedo, "Application of MPC strategies to the management of complex networks of the urban water cycle," IEEE Control Systems Magazine, vol. 33, no. 1, pp. 15-41, 2013. 